\title{
sciendo
}

\section{Smoking as a bad habit: a behavioral economics perspective}

\author{
Mădălina-Elena ȘTEFĂNEȚ \\ The Bucharest University of Economic Studies, Bucharest, Romania \\ mada.stefanet@gmail.com \\ Simona GRĂDINARU \\ The Bucharest University of Economic Studies, Bucharest, Romania \\ simonaegradinaru@gmail.com \\ Alexandru COȘER \\ The Bucharest University of Economic Studies, Bucharest, Romania \\ alexandru.coser@gmail.com \\ Stelian STANCU \\ Department of Economic Informatics and Cybernetics, \\ Bucharest University of Economic Studies \\ stelian stancu@yahoo.com
}

\begin{abstract}
Tobacco consumption is a problem of both health and economic interest nowadays. According to recent studies conducted by the European Commission approximate 700,000 deaths per year are caused by smoking. For this reason, the European Commission frequently conducts a survey in order to monitor the attitude towards tobacco addiction. Smoking addiction changes due to differentfactors such as budget, time or entourage. The evolution in time of these factors and the consumers' preferences is studied using behavioral economics based on a small group of respondents. Through a survey, over 500 persons were asked to choose their preference for cigarettes characteristics. We employ correspondence analysis using combinations of age, type of cigarette, number of cigarettes smoked per day and nicotine concertation to see the type of responses the consumers' have according to their habit. Moreover, we made a 5 persons selection from the initial group and we observed their behavior for 9 months period of time. The consumers were asked to classify a set of packages according to their preferences and we applied conjoint analysis in order to determine how or if the initial preferences change. Furthermore, we explain the changes in behavior by taking into account the nowadays global impetus towards a healthier lifestyle. The results provided allow to emphasize the role of a strong analysis for each single target consumer's behavior as this is one of the main roles of Behavioral Economics.
\end{abstract}

Keywords: behavioral economics, correspondence analysis, conjoint analysis, data mining, smoking addiction.

\section{Introduction}

Smoking addiction is an important health issue and a global problem (Petry \& Bickel, 1998). To address this problem, many restrictions were adopted at an international level, such as the prohibition of smoking inside public places (Petry, 2001). Tobacco remains one of the most profitable industries, despite the restrictions against smoking, as well as its health impact. For example, in United States of America approximate 400000 people die due to 
smoking every year. Comparing the previous number with other drugs that cause death, smoking is the deadliest drug.

The European Commission regularly conducts different studies in order to observe the evolution of population addiction to nicotine. A recent study showed that the number of people who smoke decreased by six percentage between 2006 and 2017. There is a great behavioral diversity across countries among those persons who used to smoke and quit. The proportion of respondents who have never smoked has risen by six percentage points since the 2006 survey. In general, the rate of change slowed down in consecutive surveys: in all but two cases the change since December 2014 is no more than five percentage points. Among active smokers, over nine in ten (91\%) smokers consume on daily bases tobacco products. Among cigarettes' smokers, the average number of cigars is 13.7. From the respondents over $44 \%$ smokes between 11 and 20 cigarettes and just 29\% of people smoke between 6 and 10 cigarettes. Important to mention that only $9 \%$ of respondents smoke 21 cigarettes or more (European commission report, 2017).

According to economic theory, a consumer is addicted to a product if its current usage is based on a previous period of abuse. Cigarettes addiction takes a physical form, based on nicotine effect on the brain and a psychological one, in association with the gesture. To define addiction, there are three concepts that are used: tolerance, resistance and quitting. When a person is developing tolerance for a substance is because he has a background of heavy usage of it and results in a deviated utility in the actual level of usage. In other words, the body gets used with the substance and a bigger quantity is needed for satisfying that specific need. The definition of resistance is connected to the benefits of using that drug such as pleasure, nicotine effects, physic-social effects and gesture. Quitting is characterized by the body reaction to the lack of nicotine only after the customer stops smoking or reduces the number of cigarettes. Symptoms associated with this part of the process are hypertension, anxiety, nausea and irritability.

Cigarettes are made from addictive substances. In order to understand the decision of a smoker is essential to identify the factors that stimulate the consumer behavior. Cigarettes influence the health of both smokers and non-smokers (on this category the effects will not manifest immediately, but in the future), generating an addiction. Heavy users typically have a pattern of starting to smoke in their childhood, more frequently as teenagers.

In order to comprehend the smoking patterns, we study how people addicted to nicotine change their smoking behavior over time. People's behavior suffers many changes due to population growth, risk, stress and the evolution of technology (Petry, 2001). Behavioral economics is the science that analyses the influence of various factors on how the customer takes the buying decision and changes it over time. We focus on behavioral economics concepts, namely on how the preference and addiction for bad habits changes in time due to the influence of the entourage. Within behavioral economics perspective, people described by traditional models cannot be found in real life, therefore real humans misbehave. This paper aims to determine how people try to give up smoking or what changes occur in their behavior of smoking due to their entourage, employing a behavioral economics framework. The study contains three phases, as following: first, we conducted a survey among 500 people with ages between 18 and 24 years old. In the second phase, based on the selected attributes we performed a correspondence analysis in order to identify the relationships between variables. In the third part, we followed for a year the individual behavior of 5 smokers out of 410 people. 


\section{Literature review}

Behavioral economics explains the contribution of temporary cut-off of the cigarette's consumption. Furthermore, recent studies of neuro-economics have noticed some characteristics of the drug users that could explain why these people put their life in danger. One needs to see the consumer behavior evolution in time and also to understand which factors cause changes to his choices in order to be able to understand the consumer behavior. Behavioral economics is an emergent phenomenon that advanced in time due to the evolution of both the market and customer. Miller (2009), one of the greatest contemporary psychologists, pays a great attention to this evolution from prehistoric people until now. He emphasizes the DNA changes that influence how human brain works and also its influence on the consumer behavior.

Correspondence analysis is an exploratory technique that allows the graphical representation of multi-variance categorical data. This technique is helpful in highlighting the patterns and relationships that form between data sets (Hair et al., 1995). Because it works well with small data sets and requires minimal assumptions about the measurement properties of data, this technique is well suited to social sciences and marketing research (Hoffman \& Franke, 1986; Herman, 1991). For an efficient implementation of correspondence analysis, there are three main requirements which need to be satisfied (Lebart et al., 1984). First implies that the contingency table should be large enough so that it could be analyzed through visual representation. Second constraint states that all variables have to be homogeneous, allowing to calculate a statistical distance between data points with a meaningful interpretation (Hoffman \& Franke, 1986, p.218). Third requirement affirms that correspondence analysis is the most appropriate technique in those cases where the structure of data is either poorly understood or unknown.

Conjoint analysis is a widely used multivariate technique in marketing research, which allows to determine the factors that have an influence in customers choices for different products or services (Bonilla, 2010). Conjoint analysis has been developed in the 70's when Paul E. Green and Vithala Rao made one of the first iterations of a full-profile approach (Orme, 2014). Conjoint analysis could be also perceived as a decomposition method which considers that every product or service can be 'broken down' into attributes. Therefore, this technique studies the preference for the combination of variations of such attributes (Kuzmanovic, 2008), which should cover all the possibilities for the product and should be orthogonal to each other (Orme, 2014). Furthermore, it is important to stress the utility and importance of these attributes (Levy, 1995). The utility of an attribute represents the relative importance of the attribute and is a numerical expression of the value given by consumers to that specific attribute. A low value indicates a low utility and correspondingly a high value indicates a high utility of the attribute. The importance of an attribute is assessed by calculating the difference between the highest and the lowest utility values. To sum up, conjoint analysis aims to satisfy the specific needs of customers by modeling their purchasing decisions as well as conducting specific experiments on them (Kwadzo et al., 2013; Levy, 1995).

\section{Methodology}

Correspondence analysis (CA) is a statistical technique which provides a graphical representation of the data structure of the cross tables in order to highlight the mechanisms 
underlying them. CA is used when we want to "let the data speak for itself", because it is presented as a model-free technique (Greenacre, 1999). When using it, no assumptions are made about the distribution of the variables in the contingency table to be studied. There are some debates about the definition of CA as 'model-free' and therefore we will be using CA as considered a non-linear projection of the data that can be optimal in terms of different criteria.

Most commonly used criteria in correspondence analysis are least squares, maximum likelihood or generalized least squares. Choosing a graphical representation for CA helps emphasizing certain associations between the rows and the columns variables. It is important to note that this analysis requires that all relevant categories are included and that the entries in the data matrix are nonnegative. Moreover, since it does not support the testing hypothesis, it cannot be used to test the significance of the relationships between the variables (Greenacre, 1999).

If one can form relationships between the variables, it is useful to choose only a subset. By doing so, a more simple and clear structure of the information will be achieved, even though certain data has to be disregarded. This will lead to a much clearer representation of data into a lower representational space (Redfern, 2012). One can define the mass of a data point which represents the ratio between the data point total and the total sample size and the quality of a data point indicates how well the data point is represented by the graph. The quality of the data points is connected to how well the extracted variables represent it and is characterized by a number which ranges from 0 (completely unrepresentative) to 1 (completely representative) (Redfern, 2012).

Conjoint analysis is a market research tool used to develop a more efficient version of the existing product. This technique is described as a decomposition method used for estimating people's preferences for different attributes of a product (Orme, 2014). The variables taken into consideration in conjoint analysis are called attributes. Moreover, based on the variations of each attribute (number of cigarettes, dose of nicotine, type of cigars), the according levels are defined (normal cigar, electronic cigarette or heat-not-burn product). The resulting combination of attributes and levels are called profiles. Participants in the study are asked to rank these profiles from the most wanted to the least desired profile. The next step in conjoint analysis is to calculate for each level the function of utility based on respondent choices, which defines the importance value, illustrating the impact of an attribute on each person choices.

Conjoint analysis can take three forms: traditional, adaptive and choice-based. For this study we employ a traditional conjoint analysis approach, which is also known as the "full profile approach" and involves providing respondents with full profiles, meaning that they contain levels for each attribute analyzed (Orme, 2014). Preference measured by the traditional conjoint is calculated using the following formula: $Y=b_{0}+\sum_{k=1}^{n} b_{k}\left(x_{k}\right)$, where $b_{0}$ represents the base case.

In this paper we set to answer these questions through the use of conjoint analysis: what attributes of the cigarettes are important for smokers and what importance have these attributes for the subjects. It is a priority in the analysis of consumer preferences to offer the participants different alternatives of products or services that may differ according to various attributes. These alternative options are obtained from exploratory fractional factor designs. A survey composed of product options is performed using a statistically designed tool. Multiple hypothetical alternatives of a product are presented to respondents, and these 
alternatives vary depending on their characteristics. Subjects are asked to rank products by their preferential order. In the analysis of consumer preferences, it is important that he has a wide range of products that differ by their attributes from that he can choose from. Each profile describes a product by a different combination of all the factors of interest.

\section{Data description}

The data used in this study was collected through an online survey in which people were asked to provide their age, sex and their preference for type of cigar (normal cigars, electronic cigar or heat-not-burn product), dose of nicotine and number of cigars smoked per day. 520 people answered, out of which 410 were smokers. We note that most of them were aged between 18 and 24 years (371 responses), followed by another group aged between 24-30 (86 responses), as displayed in Figure 1. The remaining age categories are not significant for the targeted group: 27 people aged between 30 and 40 years, 23 minors and 12 persons that are older that 40 years old.

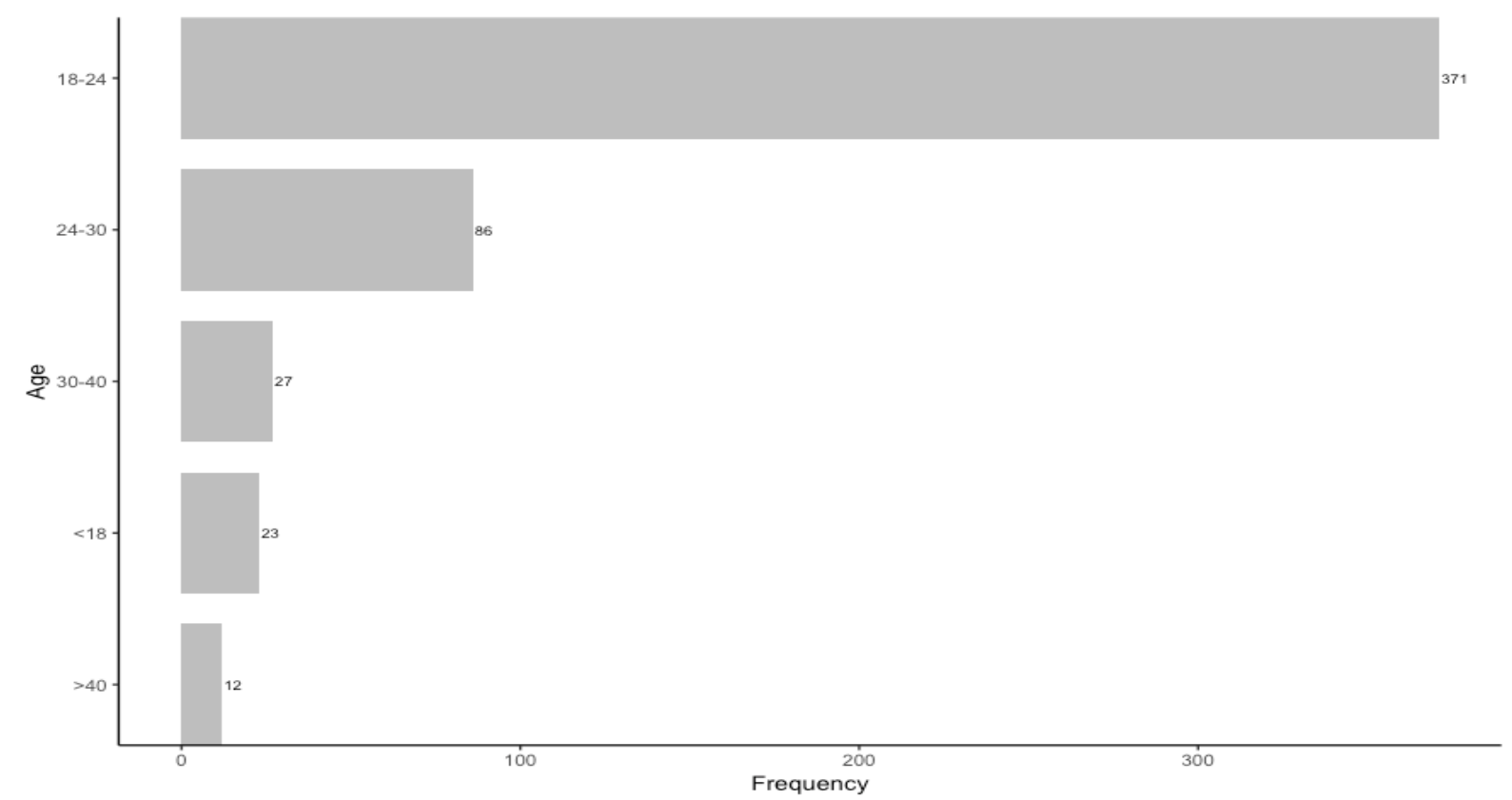

Figure 1. Age representation of respondents

Source: Authors' own research in RStudio 1.1.463

In current study we pay an essential role on the preferred type of cigars. The respondents were given the option to choose from three possibilities: normal cigars, heatnot-burn products and electronic cigarettes. The most preferred by the respondents were cigars, followed by heat not burn products, chosen by 61 people and only 6 persons smoke ecigars. (see Figure 2). The small number of people that chose e-cigars is caused by the age of the group chosen, since many people over 30 years are trying to quit smoking by choosing electronic cigars as a replacement. From another point of view, the preference for normal cigars rather that the other two options can be explained by the initial costs of an HNB product, approximate between 50 and 100 euros for a e-cigar, depending on product characteristics. 


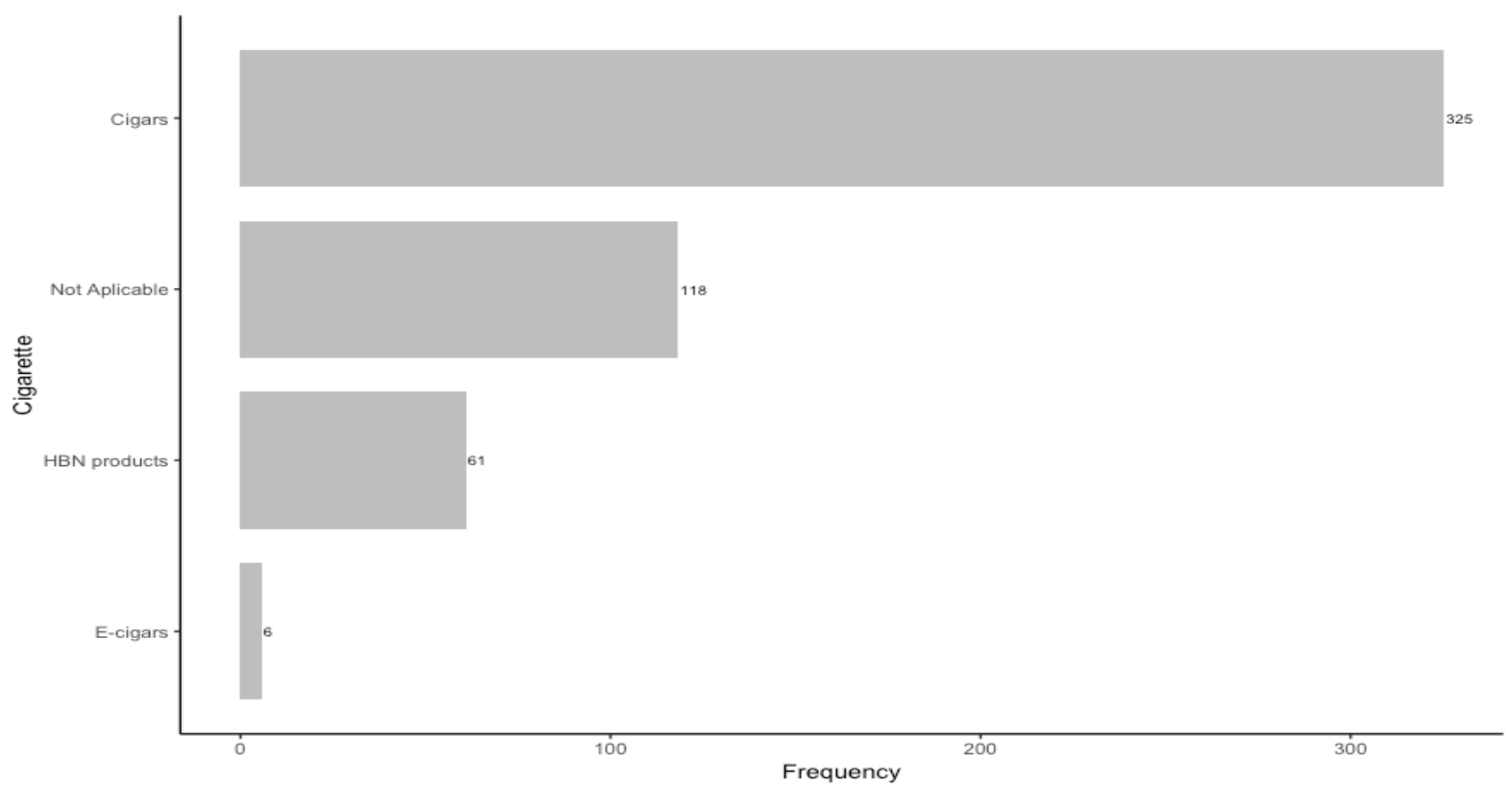

Figure 2. Type of preferred cigars

Source: Authors' own research in RStudio 1.1.463

An important aspect of smoking addiction is the repetitive gesture with the hand. For this reason, we were interested in the number of cigars smoked per day. We remark that most people smoke less than a package of cigars per day, while 33 people smoke more that 20 cigars per day. From the selected respondents, 152 said that they smoke more than half a package of cigars per day and 113 smoke close to half a package. Only 65 smoke less than 5 cigars per day (only occasionally, when they socialize) indicating that the addiction to nicotine can be ruled out from this context.

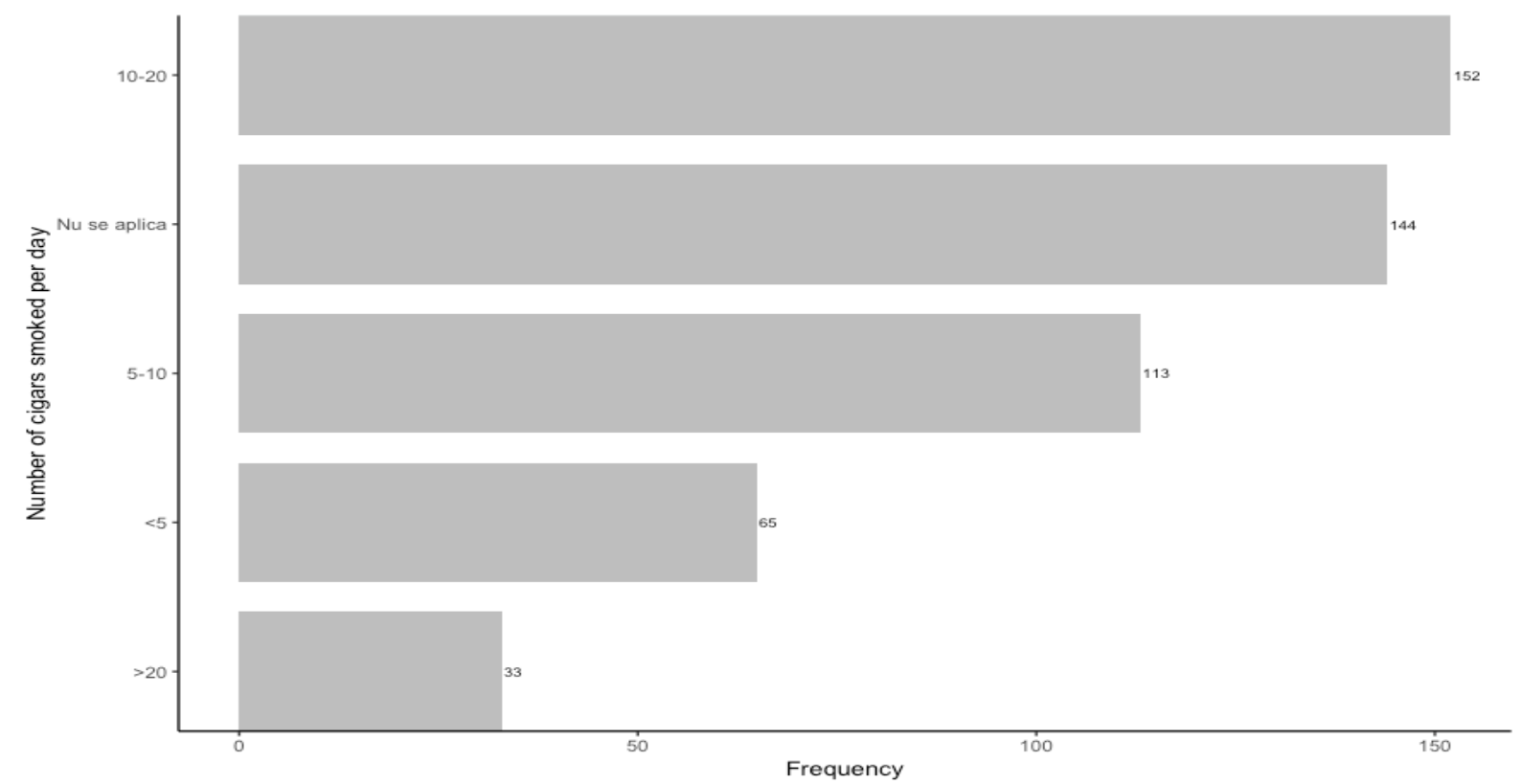

Figure 3. Number of cigars smoked per day

Source: Authors' own research in RStudio 1.1.463 
The dose is a very important part of the addiction itself, as in any other type of drug addiction. Smoking is highly influenced by the dose of nicotine inhaled. For this particular reason, people were asked to indicate the number of milligrams of nicotine in their cigars. We remarked that most of them, 283 people, chosen $0.4-0.8 \mathrm{mg}$ of nicotine. In Romania the flavored cigars have around 0.6 milligrams nicotine concentration, sustaining the above. Only 88 people chose $0.1-0.4 \mathrm{mg}$ of nicotine and 55 people chose $0.8-1.0 \mathrm{mg}$ nicotine. Only a few brands from the Romanian market have a such a high concentration of nicotine, supporting the low number of people preferring it.

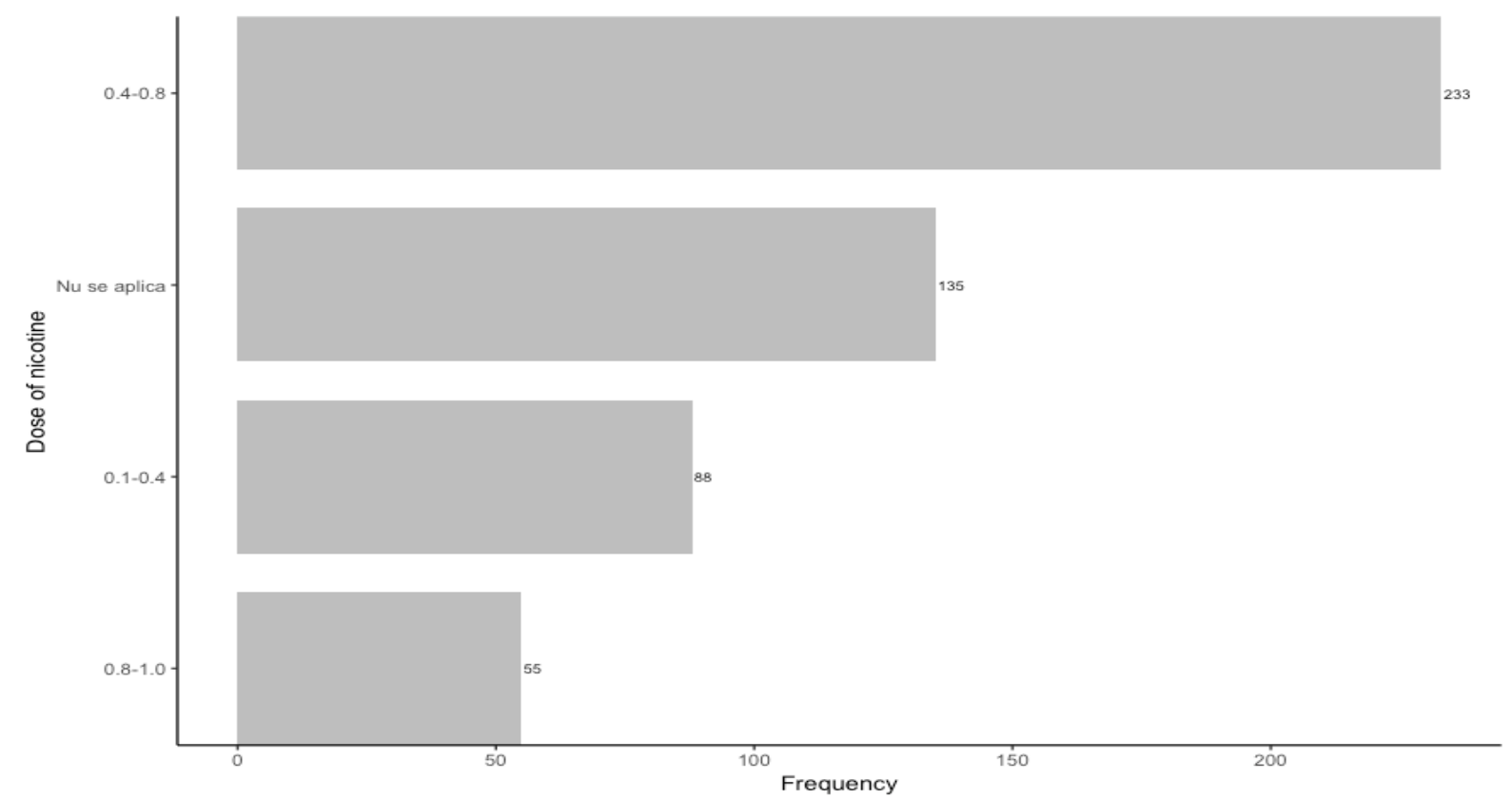

Figure 4. Representation of the preferred dose of nicotine

Source: Authors' own research in RStudio 1.1.463

\section{Results and discussions Correspondence analysis}

A correspondence analysis was applied for the contingency table containing the age and the number of cigars smoked per day. First two dimensions gather $95.1 \%$ of the original information, confirming that there are not too many insights missed by representing the data into the new subspace. The graphical representation from Figure 5 illustrates that categories are not nearby the origin, suggesting how discriminating they are from each other. 


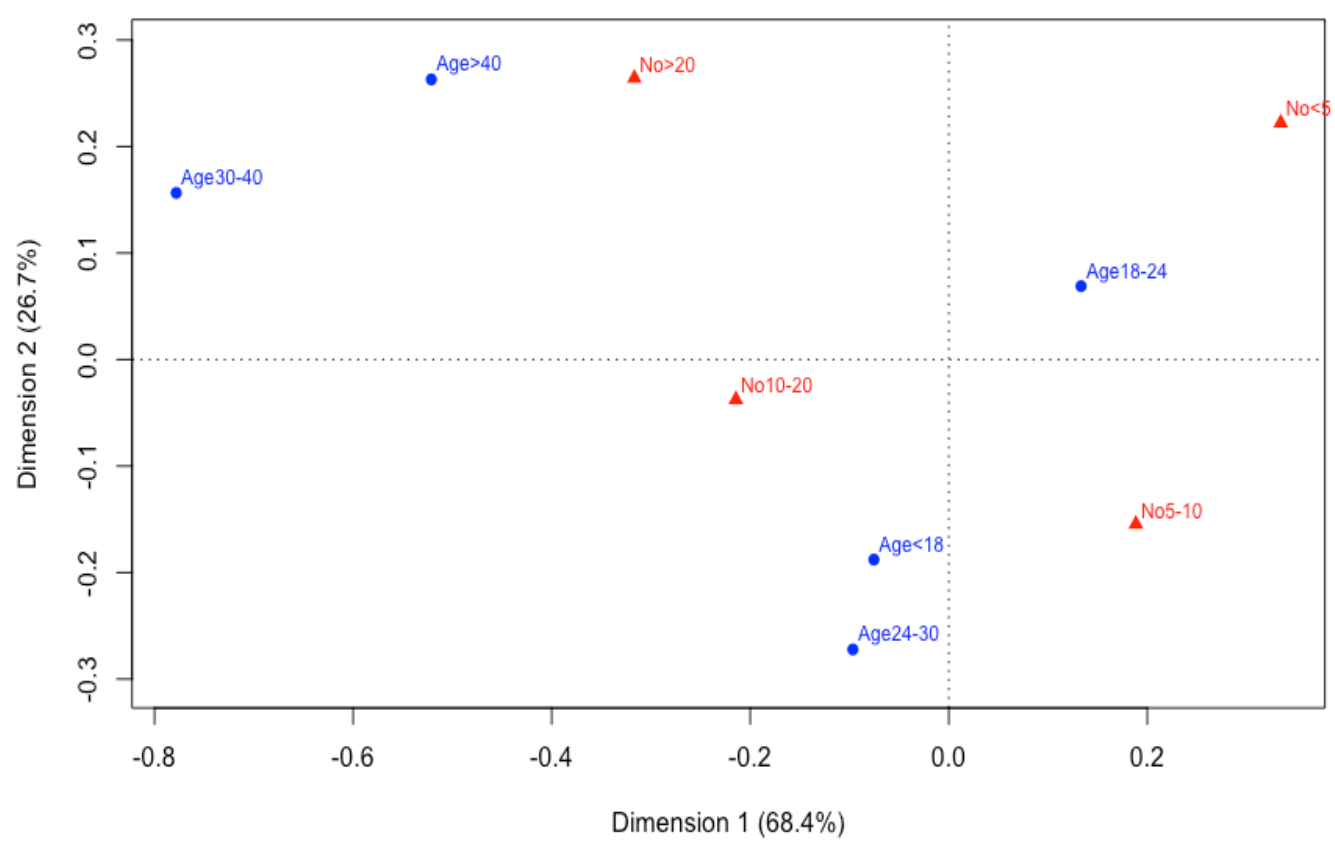

Figure 5. Correspondence analysis between age and number of cigars smoked per day

Source: Authors' own research in RStudio 1.1.463

As also remarked from the contingency table, the 18-24 age category contains most people that are smoking, while the number of cigars smoked on a daily basis doesn't matter that much for them. Same conclusion is provided by the graph, where Age 18-24 is equally distant from the first three categories of daily cigars consumption and a bit more apart from the heavy smoking category.

On the upper left side of the graphs, age categories 30-40 and above 40 are represented, opposite to 18-24 and less then 5 cigars per day, revealing a negative relationship between them. People above 30 years either don't smoke (being less likely to consume less than 5 cigars per day), either they heavily smoke 10-20 or even more than 20 cigars daily. An interested thing to observe here is that while 30-40 years people are equally distant to 10-20 and $>20$ as being indifferent to one of these categories, $40+$ aged persons are much closer to being heavily smokers (20+ cigars per day), which may be reasonable if they face high amounts of stress on a daily basis.

Age categories under 18 and 24-30 behave quite similar, by being equally distant from smoking categories 5-10 and 10-20. These persons are also equally distant from the extreme smoking categories, suggesting that there is a low likelihood for them to be associated with those ones. Therefore, they are not non-smokers and not heavily smokers, so they are somewhere in between, smoking around 5 to 20 cigars on a daily basis. 


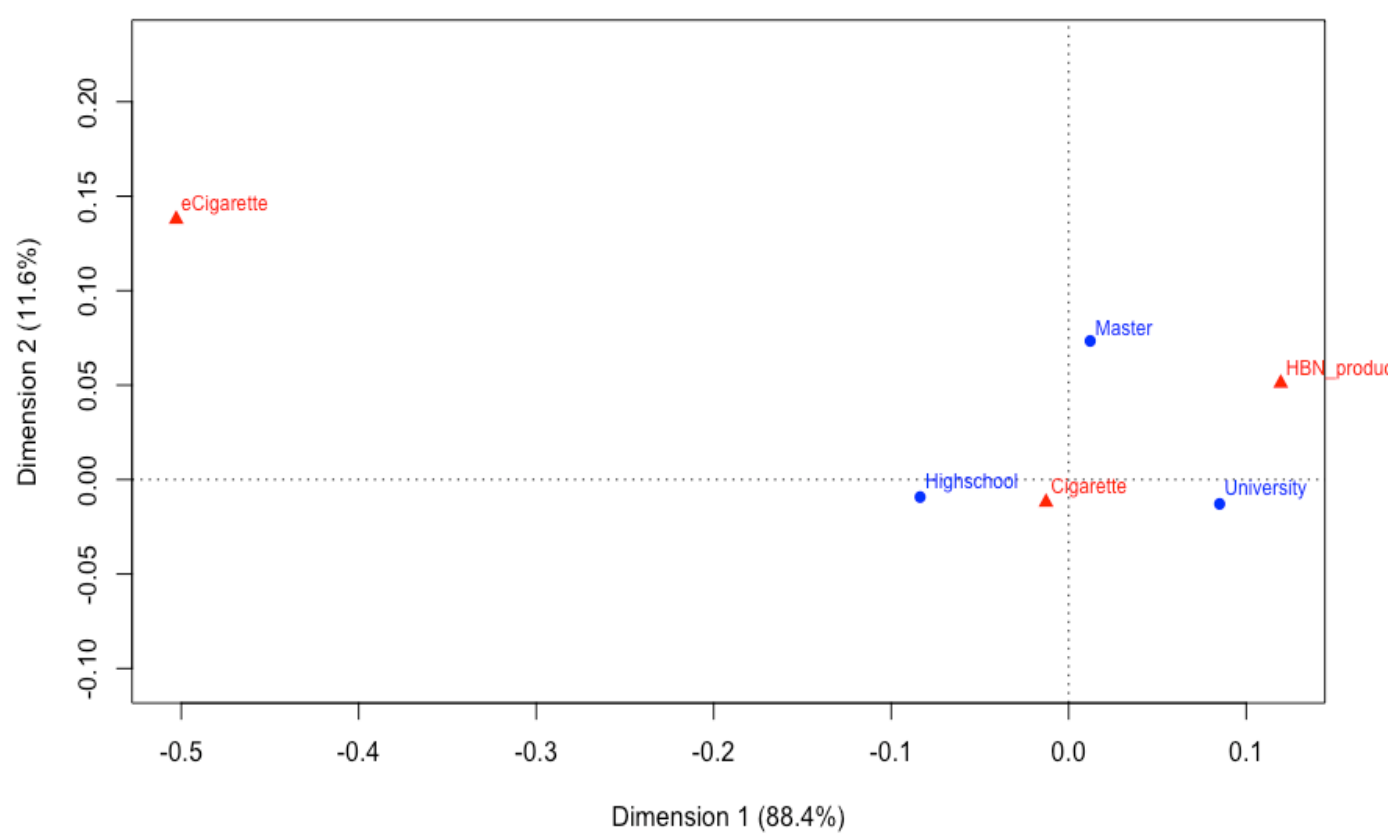

Figure 6. Correspondence analysis between education and type of cigars preferred Source: Authors' own research in RStudio 1.1.463

We applied another correspondence analysis between education and types of cigars, highlighted in the figure above (Figure 6). From the contingency table we remark that cigarettes are very frequently used by everyone and therefore, his category does not offer a good discrimination among the education types, determining its position on the graph to be nearby origin. E-cigarette products are not so popular among the respondents, driving them to be represented on the top left part of the graph, which is not surprisingly due to their elevated cost. Although this type of cigars is far away from all other categories, the closer association could be with high school smokers, but their preference is more likely to be the other categories included here. We remark that HNB products are consumed by people with higher education, with no difference between College and Master's degree. This may occur because HNB products are more expensive or even perhaps a high-end type of cigar that high school smokers do not afford, or they just do not have any interest in consuming it. This insight would have been expected, since HNB are typically perceived as a relish for educated and wealthy people, whereas high school smokers are more likely not to have any refined preferences, since their smoking habit is related to appearing interesting in an entourage.

\section{Conjoint analysis}

For conjoint analysis an important step was to define the levels of each attribute. We identified four options for the number of cigarettes: less than 5 cigars per day, between 5 and 10 cigars smoked per day, 10-20 cigars per day and more than 20 cigars. Only two of them were more meaningful for the subjects included in conjoint analysis: 5-10 and 10-20 cigars per day. Another selected attribute was the type of cigarettes which has three levels: cigars, e-cigarette and Heat-Not-Burn products (HNB). We also included three levels of the dose of nicotine: $0.1-0.4 \mathrm{mg}, 0.4-0.8 \mathrm{mg}$ and more than $0.8 \mathrm{mg}$, out of which we selected only two of them: $0.1-0.4 \mathrm{mg}$ and $0.4-0.8 \mathrm{mg}$. 8 profiles were defined based on the previously selected levels, presented in the table below. 
Table 1. Generated set of profile for conjoint analysis

\begin{tabular}{|l|l|l|l|}
\hline Card Id & Cigarette type & Dose of nicotine (mg) & No of cigars per day \\
\hline 1 & Cigarette & $0.4-0.8$ & $10-20$ \\
\hline 2 & HNB product & $0.4-0.8$ & $5-10$ \\
\hline 3 & HNB product & $0.1-0.4$ & $10-20$ \\
\hline 4 & Cigarette & $0.1-0.4$ & $5-10$ \\
\hline 5 & e-cigarette & $0.1-0.4$ & $5-10$ \\
\hline 6 & e- cigarette & $0.4-0.8$ & $10-20$ \\
\hline 7 & Cigarette & $0.1-0.4$ & $10-20$ \\
\hline 8 & Cigarette & $0.4-0.8$ & $5-10$ \\
\hline
\end{tabular}

Source: Authors' own research.

In order to build a full profile and capture the behavioral changes of smokers, we selected 5 persons hereinafter presented as subjects $A, B, C, D$, and E. This study was prepared during nine months with an interview at every 3 months. After this analysis, the total utility function was calculated for each subject and some of the aspects that generated changes of smoking behavior were examined.

Subject A is smoking for more than 5 years. He/she usually likes normal cigars, with a dose of nicotine between $0.4-0.8 \mathrm{mg}$ and smokes approximate 20 cigarettes per day. After all the interviews, we noticed he/she pays an importance to the type of cigars smoked. Subject A tries to quit smoking and for this reason he pays a great attention to the type of cigar rather than to the dose of nicotine. During the 9 months, Subject A has changed his preference for the nicotine dose at every interview. First, he/she chooses the first profile and he/she overestimate the utility expecting a value of 0.5 while the calculated utility has an actual value of 0.27 . Three months later when he/she has to choose again, A preferred the seventh package, overestimating once more the utility, expecting 1 versus a real calculated value of 0.68 .

Subject B is smoking for over 10 years and prefers normal cigars with a dose of nicotine between $0.4-0.8$ and usually smokes up to 20 cigarettes per day, with no change in preference during time. He/She classifies the packages in the same order, at every interview, with no exception. This behavior is jointly caused by nicotine addiction and brand affinity. The most important attribute for this subject is definitely the type of cigar, followed by the number of cigarettes smoked per day, whereas the dose of nicotine that has no significant impact. Every time he/she chooses the preferred package, Subject B expects an overestimated utility of 1 and gains a utility of 0.27 .

Subject $\mathrm{C}$ smokes on a frequent basis up 20 normal cigars per day with a dose of nicotine between 0.4 and 0.8 , for approximate 5 years. For $C$, the number of cigars is not important at the beginning of the analysis. At first interview, he/she chooses the eighth profile and expects an overestimated utility of 0.5 for nicotine dose, while getting an actual 0.27 utility. However, after the first 3 months when he/she faces a stress period, the number of cigars and nicotine dose start receiving significant importance. For this reason, $\mathrm{C}$ chose to select the first profile, overestimating the utility for the nicotine dose, choosing 1, while the calculated utility is only 0.68 . Six months later, at the third interview, after overcoming the stressful period, the subject reduces the importance of nicotine and a little bit of the importance of number of cigars. However, he/he maintains his previous choice for the first profile, providing an underestimating utility for nicotine, 0 and gaining a 0.27 utility. In the end, the importance of cigarettes type is the same as in the beginning of the analysis. He/She 
selects the seventh package and expects an underestimated utility of 0 and gaining 0.68.

Subject D is smoking up to 20 cigars per day for more than seven years and he prefers normal cigarettes with nicotine between 0.4 and 0.8 . At the beginning of the study, he associates the same consideration to all three factors: number of cigars, nicotine dose and type of cigarette. After the first three months, he/she pays more attention to the type of cigars and less to number of cigars (quality over quantity). This behavioral change is encouraged due to his friends changing normal cigars with heat-not-burn product. At the 6 months interview, he/she is still trying to keep up to date with the changes in the group and increases even more the attention in cigarettes type. At the last interview, after having more experience with his change from normal cigars to heat-not-burn products, he increases the interest in factors previously ignored: number of cigars and nicotine dose.

Subject $E$ is different in his initial preference from the rest: he prefers vapor cigarette with a dose a nicotine between 0.1 and 0.4 . He pays a great interest to the type of cigars in all the nine months observed. He is careful with the number of cigars, but the social hangouts have an impact on his behavior. The dose of nicotine is influenced sometimes his friends choices, because he likes to try new flavors and these ones came in different concentrations. It is noticed that subject $\mathrm{E}$ overestimates its expectations, since he is not a nicotine consumer and therefore the consumption of this substance does not bring him any benefit.

\section{Conclusion}

Nowadays tobacco consumption is an international problem of both health and economic interest. Recent studies conducted by the European Commission shows that approximate 700,000 deaths per year in Europe are caused by smoking.

Our aim in this paper was to answer two questions: what smokers enjoy in terms of nicotine quantity and what are the preferences of cigarettes type - vapor cigarettes, normal cigarettes or hot-not-burn products, in correlation with the number of cigarettes smoked per day. Using CA we obtain that most frequently people aged between 18-24 smokes without paying so much attention to the number of cigarette. Moreover, we remark from another CA made for education category and number of cigars, that very frequently these two does not cause one another, and for this reason education does not offer a good discrimination among the number of cigarettes smoked per day. In order to observe the customer behavioral changes, we studied five people for 9 months. Subject A tried to quit smoking and he changed his preference during time in type of cigarettes and his concern in how many milligrams of nicotine have the smoked cigars. Subject $B$ has not made any changes, while subject $C$ has a smoking behavior influenced by high-peak stress periods. For subject D, his preference in smoking suffers changes according to the group of friends and their preferences. The last subject studied, E, is a non-frequent smoker and for this reason he prefers smoking electronic cigarette, providing him a small dose of nicotine, 0.1-0.4 milligrams.

Another aim of this paper was to find out the most important factors which have a significant impact on smokers' preference. We conducted a survey through 500 people to measure the impact of different factors. We proved that the most important aspect for a smoker is the type of cigarette, followed by the concentration of nicotine and lastly the number of cigarettes smoked per day.

This paper can serve as a starting point for the non-governmental organizations that promotes healthy life or even a Tobacco company. The further participants could be smokers of a certain Tobacco brand and in this way the company or the NGO can generate more 
specific commercial or advise. From another point of view, the NGO could create an application that can help people quit based on their changing behavior.

\section{References}

Bickel, W. K., Miller, M. L., Yi, R., Kowal, B. P., Lindquist, D. M., \& Pitcock, J. A. (2007). Behavioral and neuroeconomics of drug addiction: Competing neural systems and temporal discounting processes. Drug and Alcohol Dependence, 90. doi:10.1016/j.drugalcdep.2006.09.016

DG.COMM.D-4. (n.d.). Retrieved from http://ec.europa.eu/commfrontoffice/publicopinion/index.cfm/Survey/getSurveyD etail/instruments/SPECIAL/surveyKy/2146

Greenacre, M. J. (1999). Correspondence analysis in the social sciences: Recent developments and applications. San Diego, Calif.: Academic Press.

Hoffman, D., Franke, G. (1986). Correspondence Analysis: Graphical Representation of Categorical Data in Marketing Research. Journal of Marketing Research, 23(3), 213227.

Kahneman, D. (2015). Thinking, fast and slow. New York: Farrar, Straus and Giroux.

Kuzmanovic, M. (2008). The nonstandard algorithm for constructing efficient conjoint experimental designs. Yugoslav Journal of Operations Research, 18(1), 63-74. doi:10.2298/yjor0801063k

Orme, B. K. (2014). Getting started with conjoint analysis: Strategies for product design and pricing research. Glendale, CA, USA: Research LLC.

Perkins, K., Donny, E., \& Caggiula, A. (1999). Sex differences in nicotine effects and selfadministration: Review of human and animal evidence. Nicotine \& Tobacco Research,1(4), 301-315. doi:10.1080/14622299050011431

Petry, N., \& Bickel, W. (1998). A Behavioral Economic Analysis of Polydrug Abuse in Heroin Addicts. doi:10.3386/w6415

Petry, N. M. (2001). The effects of housing costs on polydrug abuse patterns: A comparison of heroin, cocaine, and alcohol abusers. Experimental and Clinical

Psychopharmacology,9(1), 47-58. doi:10.1037//1064-1297.9.1.47

Petry, N. M. (2001). A behavioral economic analysis of polydrug abuse in alcoholics: Asymmetrical substitution of alcohol and cocaine. Drug and Alcohol Dependence,62(1), 31-39. doi:10.1016/s0376-8716(00)00157-5

Redfern, N. (2012). Genre trends at the US box office, 1991 to 2010. European Journal of American Culture,31(2), 145-167. doi:10.1386/ejac.31.2.145_1

RStudio Team (2016). RStudio: Integrated Development for R. RStudio, Inc., Boston, MA URL http://www.rstudio.com/.

Townsend, J., Roderick, P., \& Cooper, J. (1994). Cigarette smoking by socioeconomic group, sex, and age: Effects of price, income, and health publicity. Bmj,309(6959), 923-927. doi:10.1136/bmj.309.6959.923

Young, C., \& Skorga, P. (2010). Reduction Versus Abrupt Cessation in Smokers Who Want to Quit: A Review Summary. Public Health Nursing,28(1), 54-56. doi:10.1111/j.15251446.2010.00920.x 